\title{
Acute toxicities after extremely hypofractionated radiotherapy for prostate cancer: lessons from HYPO-RT-PC and PACE-B
}

\author{
Jesus E. Juarez, Amar U. Kishan \\ Department of Radiation Oncology, University of California, Los Angeles, CA, USA \\ Correspondence to: Amar U. Kishan, MD. University of California, 200 UCLA Medical Plaza, Suite B265, Los Angeles, CA 90095, USA. \\ Email: aukishan@mednet.ucla.edu. \\ Provenance and Peer Review: This article was commissioned and reviewed by the Guest Section Editor Xiao Li, MD (Department of Urology, Jiangsu \\ Cancer Hospital, Jiangsu Institute of Cancer Research, Nanjing Medical University Affiliated Cancer Hospital, Nanjing, China). \\ Comment on: Brand DH, Tree AC, Ostler P, et al. Intensity-modulated fractionated radiotherapy versus stereotactic body radiotherapy for \\ prostate cancer (PACE-B): acute toxicity findings from an international, randomised, open-label, phase 3, non-inferiority trial. Lancet Oncol \\ 2019;20:1531-43.
}

Submitted May 11, 2020. Accepted for publication Jun 30, 2020.

doi: $10.21037 /$ tcr-20-2061

View this article at: http://dx.doi.org/10.21037/tcr-20-2061

Although prostate cancer $(\mathrm{PCa})$ is the most common malignancy in men, accounting for nearly 1 in 5 new cancer diagnoses in the USA, it has the highest fiveyear relative survival rate (1). Most men diagnosed with PCa have localized disease, which may present with low-, intermediate-, or high-risk biochemical relapse as defined by the National Comprehensive Cancer Network (NCCN) (2). At the localized stage, various management options are available for $\mathrm{PCa}$, including definitive external beam radiotherapy (RT), radical prostatectomy, brachytherapy, and active surveillance (for low-risk and favorable intermediate-risk disease).

In the traditional course of RT, called conventionally fractionated RT (CF-RT), small fractions of radiation (1.8 to 2.0 Gy each) are delivered over 39 to 45 treatment sessions. This form of radiation has been the historical standard for many different types of cancers, with the delivery of a low dose per day thought to optimize the balance between tumor cell death and normal tissue damage. As data began to mount that suggested that PCa may exhibit an enhanced sensitivity to higher dose per fraction (3), considerable research effort was focused on improving the therapeutic ratio and optimizing patient convenience by delivering a higher dose per day. Initially, this was explored in the context of moderately hypofractionated RT (MHF$\mathrm{RT}$ ), in which the fractional dose of radiation ranged from 2.4-3.4 Gy over 20 to 30 treatment sessions. Three large randomized clinical trials demonstrated that the efficacy and safety of MHF-RT was comparable to that of CF-RT (4-6). Though most superiority trials failed to show superiority in oncologic outcome-with one exception, the trial by Hoffman et al. (7)-MHF-RT became widely considered to be a standard approach for localized PCa by late 2018 (8).

Ultra-hypofractionated radiotherapy (UF-RT) is a regimen that delivers doses of $>5$ Gy per fraction. Stereotactic body radiotherapy (SBRT, also known as stereotactic ablative radiotherapy) is a specific form of UFRT that utilizes sophisticated delivery methods to precisely deliver radiation in five or fewer treatment sessions. Given early efficacy and safety reports, in 2014 SBRT was first included in the NCCN guidelines as a potential option for patients with localized disease; however, with one caveat that "longer follow-up and prospective multi-institutional data are required to evaluate longer-term results" (9). The most recent 2020 NCCN guidelines indicates SBRT is now a standard of care option in the treatment of clinically localized $\mathrm{PCa}$, reflecting the growing evidence in support of this modality over the intervening years.

One of the landmark clinical trials known as HYPO-RTPC was published in 2019 and compared 5-year failurefree survival (FFS) in patients with intermediate- and highrisk PCa who received either UF-RT or CF-RT. UFRT treated patients received 42.7 Gy in seven fractions of 6.1 Gy over the span of 2.5 weeks, while CF-RT treated 
patients received 79 Gy in 38 fractions of 2 Gy (10). This trial, which enrolled 1,200 men across 12 Swedish centers from 2005-2015, was originally designed to demonstrate a $10 \%$ absolute improvement in 5 -year FFS from $70 \%$ to $80 \%$ among men with intermediate- and high-risk disease. Ultimately, $89 \%$ of enrolled men with intermediate-risk disease had a higher FFS than anticipated at an interim analysis, prompting the trial to be amended to demonstrate the non-inferiority of UF-RT.

The HYPO-RT-PC trial had a median follow-up time of 5 years and 5 -year FFS rates of $84 \%$ in both groups, with an adjusted hazard ratio of $1.002(\mathrm{P}=0.99)$, confirming UF-RT oncologic non-inferiority. Acute patient-reported outcomes on Prostate Cancer Symptom Scale (PCSS) questionnaire revealed urinary and bowel scales with UF-RT were significantly worse at treatment completion and remained significantly worse three months after treatment. Late grade genitourinary $(\mathrm{GU})$ and gastrointestinal (GI) toxicities were reported on the Radiation Therapy Oncology Group (RTOG) scale. The prevalence of late grade $\geq 2 \mathrm{GU}$ toxicity at one year was significantly greater with UF-RT arm ( $6 \%$ vs. $2 \%, \mathrm{P}=0.0037$ ), and urinary PCSS scores were significantly worse. Nevertheless, in UF-RT and CF-RT treated patients, the 5 -year cumulative incidence of grade $\geq 2$ GU toxicity (18\% vs. $17 \%)$ and GI toxicity (10\% vs. $10 \%)$ were similar. The 5 -year cumulative incidence of grade $\geq 3$ GU and GI toxicities were low across both arms (4.2\% vs. $4.7 \%$ for GU and $1.7 \%$ vs. $1.9 \%$ for GI). Furthermore, erectile function worsened decreasing from $70 \%$ at baseline to $35 \%$ at 5 years in both arms.

Notably, the radiation planning technique used for the majority of the patients was three-dimensional conformal radiotherapy, rather than the safer and newer intensity modulated radiotherapy (IMRT) (11). Moreover, while $90 \%$ of patients did have implanted fiducial markers to help mitigate the impact of prostate motion between fractions, the planning margins used were $7 \mathrm{~mm}$ isotropically; this would be considered large by contemporary standards (i.e., a large volume of tissue was exposed to radiation). Consequently, patients enrolled on the HYPO-RT-PC trial may have been subjected to higher absolute rates of toxicity than what would be expected with modern treatment planning and delivery. Thus, the difference in toxicity for patients receiving CF-RT and UF-RT remains unclear.

Overall, the HYPO-RT-PC trial then appears to be much more a test of the radiobiological premise underlying UF-RT, rather than a direct assessment of modern and more precise SBRT. The oncologic non-inferiority and the lack of clear differences in late toxicity suggest that these radiobiological considerations are in fact valid. Nevertheless, other studies will be required to evaluate whether differences in technical aspects of radiation planning and delivery will affect efficacy and toxicity in a fractionation-dependent manner.

The PACE-B trial is one such trial. This study enrolled 874 men with low- or intermediate-risk PCa in the United Kingdom and Canada between 2012 and 2018 and directly compared modern SBRT with a CF-RT or MHF-RT control arms. The SBRT arm delivered 36.25 Gy either consecutively in five fractions of 7.25 Gy $(20.7 \%)$ or over the span of $\sim 2$ weeks $(79.3 \%$ ), while the CF-RT control arm delivered 78 Gy in 39 fractions of 2 Gy each (31\%) and the MHF-RT control arm delivered 62 Gy in 20 fractions of 3.1 Gy each (69\%).(12) The authors designed PACE-B to demonstrate the non-inferiority of SBRT (margin set at $6 \%$ ) with respect to freedom from biochemical or clinical failure at five years.

In a pre-specified substudy, the authors evaluated acute physician-scored toxicity on the RTOG scale. This analysis aimed to exclude SBRT-treated patients who had a $10 \%$ increase from $25 \%$ and a $11 \%$ increase from $40 \%$ in RTOG grade $\geq 2$ GI toxicity and GU toxicity, respectively. The results of this study revealed that the rates of worst RTOG grade $\geq 2$ GI and GU toxicity typically occurred 4-6 weeks after treatment completion, and the rates did not differ significantly between the SBRT and control arms (9.3\% vs. $13.2 \%$ for GI and $20.2 \%$ vs. $26.8 \%$ for GU). By 12 weeks, similar rates of RTOG grade $\geq 2$ GI and GU toxicities exceeding baseline had dropped in both SBRT and control arms ( $1.7 \%$ vs. $0.5 \%$ for GI and $5 \%$ vs. $3.8 \%$ for GU). In addition, no differences were identified between SBRT and control arms in Expanded Prostate Cancer Index Composite (EPIC) based evaluation of patient-reported toxicity (bowel, urinary, and sexual bother), or in the Common Terminology Criteria for Adverse Events (CTCAE) scale acute grade $\geq 2$ GU toxicity. However, SBRT-treated patients did experience significantly worse acute CTCAE grade $\geq 2$ GI toxicity (15.2\% vs. 8\%, $\mathrm{P}=0.011)$, exceeding baseline, though this difference disappeared by 12 weeks. The difference in acute GI toxicity appeared to have been driven by grade 2 diarrhea $(6.5 \%$ vs. $1.4 \%)$ and proctitis $(5.7 \% v s$. $2.5 \%)$ at the $4-6$ weeks timepoint. Of note, the time from initiation of radiation to when CTCAE was reported was significantly different depending on the trail arm, whereas RTOG toxicity was assessed at every visit and may have more comprehensively captured toxicity patterns. 
It is possible that the quantitative differences in acute grade $\geq 2$ RTOG GU toxicity rates in PACE-B versus HYPO-RT-PC (20.8\% vs. 28\%) might relate to the fact that PACE-B required IMRT planning, whereas only $20 \%$ of patients in the HYPO-RT-PC had IMRT planning. However, even the acute toxicity rates from the PACE-B trial might be evaluated critically. Only $73 \%$ of patients on PACE-B had implanted fiducials for inter-fraction monitoring, and only $41.7 \%$ of patients on PACE-B had intra-fraction motion monitoring. While there is minimal high-level evidence suggesting that more rigorous motion management strategies are needed, it is conceivable that more sophisticated motion management strategies could allow smaller margins around the target, thereby reducing the amount of normal tissue receiving radiation. Furthermore, the increased toxicity seen with SBRT in the PACE-B trial could be attributed to daily fractionation; given that prior studies suggest that longer intervals between SBRT fractions diminish toxicity $(13,14)$.

Both trials support SBRT as an excellent option for patients receiving radiotherapy for PCa. Longer-term results from the PACE-B will help confirm the oncologic non-inferiority of this approach, while two ongoing trials are designed to evaluate superiority of SBRT over moderate hypofractionation \{NRG GU-005 [NCT03367702] \} or surgery $\{$ PACE-A [NCT01584258]\} from a quality-oflife standpoint. Other technological advancements may further optimize quality-of-life following SBRT. One promising modality is magnetic resonance imaging (MRI)guided radiotherapy. MRI-based delivery platforms integrate a MRI scanner and linear accelerator allowing for superior quality and "real-time" imaging during treatment delivery, thereby achieving tighter treatment margins $(15,16)$. Furthermore, data from one randomized trial has demonstrated that insertion of a hydrogel spacer between the rectum and prostate reduces the rectal dose toxicity and improves quality-of-life following radiotherapy (17), thus its use in the context of SBRT may lead to further reductions in toxicity. Finally, mounting evidence suggests that severity of adverse reactions to radiation might be influenced by variations in germline DNA $(18,19)$. Preliminary data suggest that distinct germline variations may predispose patients to late toxicity after prostate-directed radiotherapy in a fractionation-dependent manner (20). Thus, biomarker panels may help identify patients who are more likely to experience significant toxicity after a particular regimen, thereby optimizing the choice of a fractionation regimen.

SBRT is a new and advanced modality that offers many benefits over conventional radiotherapy, with mounting evidence supporting its use for treating clinically localized PCa. Current research being conducted will help optimize SBRT to deliver personalized and targeted treatments. Ultimately, these results will guide major paradigm shifts in the way PCa is treated globally.

\section{Acknowledgments}

Funding: None.

\section{Footnote}

Conflicts of Interest: Both authors have completed the ICMJE uniform disclosure form (available at http://dx.doi. org/10.21037/tcr-20-2061). AUK reports grants and personal fees from ViewRay, Inc., personal fees from Varian Medical Systems, Inc., Intelligent Automation, Inc., other from Janssen, Inc., outside the submitted work. JEJ has no conflicts of interest to declare.

Ethical Statement: The authors are accountable for all aspects of the work in ensuring that questions related to the accuracy or integrity of any part of the work are appropriately investigated and resolved.

Open Access Statement: This is an Open Access article distributed in accordance with the Creative Commons Attribution-NonCommercial-NoDerivs 4.0 International License (CC BY-NC-ND 4.0), which permits the noncommercial replication and distribution of the article with the strict proviso that no changes or edits are made and the original work is properly cited (including links to both the formal publication through the relevant DOI and the license). See: https://creativecommons.org/licenses/by-nc-nd/4.0/.

\section{References}

1. Siegel RL, Miller KD, Jemal A. Cancer statistics, 2019. CA Cancer J Clin 2019;69:7-34.

2. Network NCC. NCCN Clinical Practice Guidelines in Oncology: Prostate Cancer. 2020. Accessed 4/15/2020. Available online: http://www.nccn.org/professionals/ physician_gls/pdf/prostate.pdf

3. Brenner DJ, Hall EJ. Fractionation and protraction for radiotherapy of prostate carcinoma. Int J Radiat Oncol Biol Phys 1999;43:1095-101.

4. Dearnaley D, Syndikus I, Mossop H, et al. Conventional 
versus hypofractionated high-dose intensity-modulated radiotherapy for prostate cancer: 5-year outcomes of the randomised, non-inferiority, phase $3 \mathrm{CHHiP}$ trial. Lancet Oncol 2016;17:1047-60.

5. Lee WR, Dignam JJ, Amin MB, et al. Randomized Phase III Noninferiority Study Comparing Two Radiotherapy Fractionation Schedules in Patients With Low-Risk Prostate Cancer. J Clin Oncol 2016;34:2325-32.

6. Catton CN, Lukka H, Gu CS, et al. Randomized Trial of a Hypofractionated Radiation Regimen for the Treatment of Localized Prostate Cancer. J Clin Oncol 2017;35:1884-90.

7. Hoffman KE, Voong KR, Levy LB, et al. Randomized Trial of Hypofractionated, Dose-Escalated, IntensityModulated Radiation Therapy (IMRT) Versus Conventionally Fractionated IMRT for Localized Prostate Cancer. J Clin Oncol 2018;36:2943-9.

8. Morgan SC, Hoffman K, Loblaw DA, et al. Hypofractionated Radiation Therapy for Localized Prostate Cancer: An ASTRO, ASCO, and AUA EvidenceBased Guideline. 2018. doi: 10.1016/j.juro.2018.10.001.

9. King CR, Freeman D, Kaplan I, et al. Stereotactic body radiotherapy for localized prostate cancer: pooled analysis from a multi-institutional consortium of prospective phase II trials. Radiother Oncol 2013;109:217-21.

10. Widmark A, Gunnlaugsson A, Beckman L, et al. Ultrahypofractionated versus conventionally fractionated radiotherapy for prostate cancer: 5-year outcomes of the HYPO-RT-PC randomised, non-inferiority, phase 3 trial. Lancet 2019;394:385-95.

11. Michalski JM, Yan Y, Watkins-Bruner D, et al. Preliminary toxicity analysis of 3-dimensional conformal radiation therapy versus intensity modulated radiation therapy on the high-dose arm of the Radiation Therapy Oncology Group 0126 prostate cancer trial. Int J Radiat Oncol Biol Phys 2013;87:932-8.

12. Brand DH, Tree AC, Ostler P, et al. Intensity-

Cite this article as: Juarez JE, Kishan AU. Acute toxicities after extremely hypofractionated radiotherapy for prostate cancer: lessons from HYPO-RT-PC and PACE-B. Transl Cancer Res 2020;9(8):4469-4472. doi: 10.21037/tcr-20-2061. modulated fractionated radiotherapy versus stereotactic body radiotherapy for prostate cancer (PACE-B): acute toxicity findings from an international, randomised, open-label, phase 3, non-inferiority trial. Lancet Oncol 2019;20:1531-43.

13. Quon HC, Ong A, Cheung P, et al. Once-weekly versus every-other-day stereotactic body radiotherapy in patients with prostate cancer (PATRIOT): A phase 2 randomized trial. Radiother Oncol 2018;127:206-12.

14. Kishan AU, Dang A, Katz AJ, et al. Long-term Outcomes of Stereotactic Body Radiotherapy for Low-Risk and Intermediate-Risk Prostate Cancer. JAMA Netw Open 2019;2:e188006.

15. Pathmanathan AU, van As NJ, Kerkmeijer LGW, et al. Magnetic Resonance Imaging-Guided Adaptive Radiation Therapy: A "Game Changer" for Prostate Treatment? Int J Radiat Oncol Biol Phys 2018;100:361-73.

16. Bruynzeel AME, Tetar SU, Oei SS, et al. A Prospective Single-Arm Phase 2 Study of Stereotactic Magnetic Resonance Guided Adaptive Radiation Therapy for Prostate Cancer: Early Toxicity Results. Int J Radiat Oncol Biol Phys 2019;105:1086-94.

17. Hamstra DA, Mariados N, Sylvester J, et al. Continued Benefit to Rectal Separation for Prostate Radiation Therapy: Final Results of a Phase III Trial. Int J Radiat Oncol Biol Phys 2017;97:976-85.

18. Kato M, Paranjape T, Müller RU, et al. The mir-34 microRNA is required for the DNA damage response in vivo in $\mathrm{C}$. elegans and in vitro in human breast cancer cells. Oncogene 2009;28:2419-24.

19. Metheetrairut C, Adams BD, Nallur S, et al. cel-mir-237 and its homologue, hsa-miR-125b, modulate the cellular response to ionizing radiation. Oncogene 2017;36:512-24.

20. Kishan AU, Ruan D, Dang AT, et al. MicroRNA-based Biomarkers predicting Long-Term Toxicity to Prostate SBRT. Int J Radiat Oncol Biol Phys 2019;105:S39-40. 